\title{
Sur une propriété des nombres naturels.
}

\author{
par Waclaw Sierpinski (à Varsovie). \\ A Mauro Picone pour son $70^{\text {me }}$ anniversaire.
}

Résumé. - L'auteur donne des conditions nécessaires et suffisantes pour qu' un nombre naturel $n$ soit un "nombre pratique", c'est-à-dire pour que tout nombre naturel $\leq n$ soit une somme de diviseurs naturels distincts du nombre $n$.

1. D'après M. A. K. SRINIVASAN on appelle un nombre naturel $n$ "nombre pratique» si tout nombre naturel $\leq n$ est une somme de diviseurs naturels distinets du nombre n. Dans "The Mathematical Gazette », 36 (1952), p. 49 , M. A. K. Saroja donne sans démonstration une condition nécessaire et suffisante pour qu' un nombre naturel $n$ soit pratique. Or, la formule qui exprime cette condition est fausse, sans doute à cause d'une faute d'impression. Un de mes élèves, M. J. Browkrn a exprimé l' hypothèse qu' on a ici le théorème suivant:

THÉORÈme 1. - Pour qu'un nombre naturel $n>1$ soit pratique, il faut et il suffit qu'il se décompose en facteurs premiers

$$
n=2 x^{\alpha_{0}} p_{1}^{\alpha_{1}} p_{2}^{\alpha_{2}} \ldots p_{k-1}^{\alpha_{k-1}}
$$

où $k, \alpha_{0}, \alpha_{1}, \ldots, \alpha_{k-1}$ sont des nombres naturels (en cas $k=0$ on a $n=2^{x_{0}}$ ) et où (dans le cas $k>1$ ) $p_{1}, p_{2}, \ldots, p_{k-1}$ sont des nombres premiers impairs, $p_{1}<p_{2}<\ldots<p_{k-1}$ et

$$
p_{i} \leq 1+\sigma\left(2^{\alpha_{0}} p_{1}^{\alpha_{1}} p_{2}^{\alpha_{2}} . . . p_{i-1}^{\alpha_{i-1}}\right) \quad \text { pour } i=1,2, \ldots, k-1,
$$

où $\sigma(l)$ désigne la somme de tous le diviseurs naturels au nombre $l$ (').

Je donnerai ici la démonstration de ce théorème qui sera basée sur le lemme suivant.

LEMмE. $-s, c, p$ et $m$ étant des nombres naturels tels que

$$
p \leq c+1 \text { et } m \leq c\left(1+p+p^{2}+\ldots+p^{s-1}\right),
$$

il existe des entiers $c_{i}(i=1,2, \ldots, s-1)$ tels que

$$
0 \leq c_{i} \leq c \text { pour } i=1,2, \ldots, s-1
$$

et

$$
m=c_{0}+c_{2} p+c_{2} p^{2}+\ldots+c_{s-1} p^{s-1}
$$

(1) Addition pendant la correction des épreuves: Cr. B. M. STEWaRT, a American Journal of Mathematics 2, 76 (1954), p. 779.785. 
Démonstration da lemme. - Le lemme est évidemment vrai pour $s=1$. Or, supposons qu'il existe des nombres naturels $s>1$ pour lesquels le lemme n'est pas vrai et soit $s$ le plus petit d'entre eux. Il existe done un nombre naturel $m$ qui est le plus petit tel qu' on a pour certains nombres naturels $p$ et $c$ les formules (3), mais qu'il n'existe pas des entiers $c_{\imath}(i=0,1, \ldots, s-1)$ pour lesquels on ait les formules (4) et (5). On a ici évidemment $m>1$ et il existe des entiers $c_{i}(i=0,1, \ldots, s-1)$ satisfaisant aux conditions (4) et tels que

$$
m-1=c_{11}+c_{1} p+c_{2} p^{2}+\ldots+c_{s-1} p^{s-1}
$$

Il ne peut ètre ioi $c_{0}=c_{1}=\ldots=c_{s-1}=c$, puisque la formule (6) serait alors incompatible avec la deuxième des formules (3).

D'après (4) un au moins des entiers $c_{n}, c_{1}, \ldots, c_{s-1}$ est $<c$ : soit $j$ le plus petit indice $\leq s-1$ tel que $c_{j}<c$. S'il était ici $j=0$, donc $c_{0}<c$, on aurait $c_{0}{ }^{\prime}=c_{0}+1 \leq c$ et $m=c_{0}{ }^{\prime}+c_{1} p+c_{2} p^{3}+\ldots+c_{s-1} p^{s-1}$, contrairement à l'hypothèse sur le nombre $m$. On a dono $j>0$ et $c_{0}=c_{1}=\ldots=c_{j-1}=c$. Donc, vu que d'après la première des formules (3) on a $c \geq p-1$, on trouve $c_{n}+c_{1} p+\ldots+c_{j-1} p^{j-1}-p^{j}+1 \geq(p-1)\left(1+p+p^{2}+\ldots+p^{j-1}\right)-p^{j}+1=0$ ef comme d'antre part on a évidemment

$$
c_{0}+c_{1} p+\ldots+c_{j-1} p^{j-1}-p^{j}+1 \leq c\left(1+p+p^{2}+\ldots+p^{j-1}\right)
$$

et $j-1<s-1$, vu la définition du nombre $s$, il existe des entiers $c_{i}^{\prime}(i=0,1, \ldots, i-1)$, tels que $0 \leq c_{i}^{\prime} \leq c$ pour $i=0,1, \ldots, j-1$ et que

$$
c_{0}+c_{1} p+\ldots+c_{j-1} p^{j-1}-p^{j}+1=c_{0}^{\prime}+c_{1}^{\prime} p+\ldots+c_{j-1}^{\prime} p^{j-1} .
$$

En posant $c_{j}^{\prime}=c_{j}+1$ nous avons donc, d'après $c_{j}<c, c_{j}^{\prime} \leq c$ et d'après (6) et (7)

$m=c_{0}^{\prime}+c_{1}^{\prime} p+\ldots+c_{j-1}^{\prime} p^{j-1}+c_{j}^{\prime} p^{j}+c_{j+1} p^{j+1}+c_{j+2} p^{j+2}+\ldots+c_{s-1} p^{s-1}$, contrairement à l'hypothèse sur le nombre $m$.

Notre lemme est done vrai pour tout nombre naturel $s$ et il se trouve ainsi démontré.

Démonstration du théorème 1 . - Soit $n>1$ un nombre naturel pratique. Il est clair que $n$ doit être un nombre pair, puisque dans le cas con. traire il serait $n>2$ et le nombre 2 n'est pas évidemment une somme de diviseurs distincts d'un nombre impair. Le nombre $n$ a done le développe. ment (1) en facteurs premiers.

Supposons maintenant que pour un nombre naturel $i \leq k-1$ la condi. tion (2) n'est pas remplie, done qu' on a

$$
p_{i}>1+\sigma\left(2^{\alpha_{0}} p_{1}^{\alpha_{1}} p_{2}^{\alpha_{2}} \ldots p_{i-1}^{\alpha_{i}-1}\right) \text {. }
$$

Les diviseurs du nombre $n$ qui ne dépassent pas le nombre $p_{i}-1$ peuvent donc ètre seulement les diviseurs du nombre $2^{x_{0}} p_{1}^{\alpha_{1}} p_{2}^{\alpha_{2}} \ldots p_{i-1}^{\alpha_{i}-1}$, et la 
somme de tous les diviseurs de ce nombre étant $\sigma\left(2^{x_{0}} p_{1}^{\alpha_{1}} p_{2}^{\alpha_{3}} \ldots p_{i-1}^{\alpha_{i-1}}\right)<p_{i}-1$, le nombre $p_{i}-1<n$ ne peut pas être une somme de diviseurs distincts du nombre $n$, contrairement à l'hypothèse que $n$ est un nombre pratique. La condition de notre théorème est ainsi nécessaire.

Nous démontrerons maintenant par l'induction (par rapport au nombre $k$ ) que si le nombre naturel $n$ remplit les conditions de notre théorème, tout nombre naturel $\leq o(n)$ est une somme des diviseurs distincts du nombre $n$.

Cela est rrai pour $k=1$, e' est-à-dire pour les nombres $n=2^{\alpha_{0}}$, puisqu'alors on a $\sigma(n)=2^{\alpha_{0}+1}-1$ et, comme on sait, tout nombre naturel $\leq 2^{\alpha_{0}+1}-1$ est une somme de termes distinets de la suite $1,2,2^{2}, \ldots 2^{\text {* }}$ (qui sont diviseurs du nombre $2^{\alpha_{0}}$ ).

Soit maintenant $k$ un nombre naturel $>1$ et supposons que notre assertion est vraie pour le nombre $k-1$, donc que tout nombre naturel $\leq \sigma\left(2 \alpha_{0} p_{1}^{z_{1}} p_{2}^{\alpha_{2}} \ldots p_{k-2}^{z_{k}-2}\right)$ est une somme de diviseurs distincts du nombre $2{ }^{\alpha_{0}} p_{1}^{\alpha_{1}} p_{2}^{\alpha_{2}} \ldots p_{k-2}^{\alpha_{k-2}}$.

Soit $m$ un nombre naturel tel que

$$
m \leq \sigma\left(2^{\alpha_{0}} p_{1}^{\alpha_{1}} p_{2}^{\alpha_{2}} \ldots p_{k-1}^{\alpha_{k-1}}\right) \text {. }
$$

Comme

$$
\sigma\left(2^{\alpha_{0}} p_{1}^{\alpha_{1}} p_{2}^{\alpha_{1}} \ldots p_{k-1}^{\alpha_{k-1}}\right)=\sigma\left(2^{\alpha_{0}} p_{1}^{\alpha_{1}} p_{2}^{\alpha_{\nu}} \ldots p_{k-2}^{\alpha_{k-2}}\right)\left(1+p_{k-1}+p_{k-1}^{2}+\ldots+p_{k-1}^{\alpha_{k-1}}\right),
$$

on a

$$
m \leq \sigma\left(2^{\alpha} p_{1}^{\alpha_{1}} p_{2}^{\alpha_{2}} \ldots p_{k-2}^{\alpha_{k-2}}\right)\left(1+p_{k-1}+p_{k-1}^{2}+\ldots p_{k-1}^{\alpha_{k-1}}\right),
$$

et, vu la formule (2) pour $i=k-1$ et d'après notre lemme pour $s=\alpha_{k-1}+1$, $c=\sigma\left(2^{x_{0}} p_{1}^{\alpha_{1}} p_{2}^{\alpha_{2}} \ldots p_{k-2}^{\alpha_{k-2}}\right)$ et $p=p_{k-1}$, on conclut $q \mathrm{u}^{\prime}$ il existe des entiers $c_{i}\left(i=0,1, \ldots, \alpha_{k-1}\right)$ tels que $0 \leq c_{i} \leq \sigma\left(2^{\alpha_{1}} p_{1}^{\alpha_{1}} \ldots p_{k-2}^{\alpha_{k-2}}\right)$ et que

$$
m=c_{0}+c_{1} p_{k-1}+c_{2} p_{k-1}^{2}+\ldots+c_{\alpha_{k-1}} p_{k-1}^{\alpha_{k-1}} .
$$

Notre assertion étant vraie, par l'hypothèse, pour le nombre $k-1$, tout nombre $c_{i}\left(i=0,1, \ldots, \alpha_{k-1}\right)$ est une somme de diviseurs distincts du nombre $2^{\alpha_{0}} p_{1}^{\alpha_{1}} p_{2}^{\alpha_{2}} \ldots p_{k-2}^{\alpha_{k-2}}$, d'où, d'après (8) on conclut tout de suite que $m$ est une somme de diviseurs distincts du nombre $2^{\alpha_{u}} p_{1}^{\alpha_{1}} p_{2}^{\alpha_{2}} . . p_{k-1}^{x_{k-1}}$. Notre assertion est ainsi vraie pour le nombre $k$.

Notre assertion, et, à plus forte raison, le théorème 1 se trouve ainsi démontré.

En particulier, d'après $5<1+\sigma\left(2^{2}\right)=8$, le nombre $100=2^{2} .5^{2}$ est pratique et tont nombre naturel $\leq \sigma(100)=217$ est une somme de diviseurs distincts du nombre 100. Pareillement 1000 est un nombre pratique et tout nombre naturel $\leq 2340$ est une somme de diviseurs distincts du nombre 1000 .

Il résulte sans peine du théorème 1 que si le nombre (1) est pratique, tout nombre naturel $m$ dont le développement en facteurs premiers est 
$m=2^{\beta_{0}} p_{1}^{\beta_{1}} p_{2}^{\beta_{2}} \ldots p_{k-1}^{\beta_{k-1}}$, où $\beta_{i}(i=0,1,2, \ldots, k-1)$ sont des nombres naturels tels que $\beta_{i} \geq \alpha_{i}$ pour $i=0,1, \ldots, k-1$, est également un nombre pratique. Il en résulte tout de suite la proposition trouvée par M. J. BRowkin que lorsqu'on multiplie un nombre pratique par un de ses diviseurs, on obtient un nombre pratique.

Il résulte aussi sans peine de notre théorème 1 que le produit de deux fou plusienrs) nombres pratiques (distincts ou non) est toujours un nombre pratique (ce qui fut aussi trouvé par M. J. Browkin).

Pour démontrer que le nombre $p_{1} p_{2} \ldots p_{n}$, ol $p_{i}$ désigne le $i$-ème nombre premier, et $n=1,2, \ldots$, est pratique, il suffit d'appliquer notre théorème et l'inégalité connue $p_{i} \leq 1+p_{1} p_{2} \ldots p_{i-1}$ pour $i=2,3, \ldots$.

Or, la proposition que pour tout $n$ naturel $>1$ le nombre $2^{n-1}\left(2^{n}-1\right)$ est pratique peut être démontrée sans peine directement. En effet, si $k$ est un nombre naturel $\leq 2^{n}-1, k$ est, comme on sait, une somme de termes distincts de la suite $1,2, \ldots, 2^{n-1}$. Or, si $2^{n}-1<k \leq 2^{n-1}\left(2^{n}-1\right)$, on a $k=\left(2^{n}-1\right) t+r$ où $t$ est un nombre naturel $\leq 2^{n-1}$ et $r$ un entier tel que $0 \leq r<2^{n}-1$, donc $t$ et $r$ sont des sommes de termes distincts de la suite $1,2, \ldots, 2^{n-1}$, d'où il résulte tout de suite la démonstration cherchée. Donc, en particulier, tout nombre parfait pair est pratique, ce qui a été signalé par M. SAROJA.

2. La notion des nombres pratiques nous conduit an probleme suivant: $n_{1}, n_{3}, \ldots, n_{k}$ étant une suite croissante donnée de nombres naturels, quelle est la condition nécessaire et suffisante pour que tout nombre naturel $n \leq n_{1}+n_{2}+\ldots+n_{k}$ soit une somme de termes distincts de cette suite?

Nous démontrerons que cette condition est $q^{\mathrm{u}}$ 'il soit

$$
n_{i} \leq 1+n_{1}+n_{2}+\ldots+n_{i-1} \text { pour } i=1,2, \ldots, k .
$$

La condition est nécessaire. En effet, s'il existe un indice $i \leq k$ tel que $n_{i}>1+n_{1}+n_{2}+\ldots+n_{i-1}$, le nombre $1+n_{1}+n_{2}+\ldots+n_{i-1}<n_{i}$ ne pourrait évidemment être une somme de termes distincts de la suite $n_{1}, n_{2}$, $\ldots, n_{k}$.

Notre condition est évidemment suffisante pour le nombre $k=1$, puisque pour $i=1$ elle donne $n_{i} \leq 1$, done $n_{i}=1$, et pour $n \leq n_{1}$ on a $n=1=n_{i}$.

Supposons maintenant que notre condition n' est pas suffisante. Il existe donc un nombre naturel $k$ qui est le plus petit ponr lequel notre condition n'est pas suffisante, et on a $k>1$. Il existe done un nombre naturel $m \leq n_{1}+n_{2}+\ldots+n_{k}$ qui n'est pas somme de termes distincts de la suite $n_{1}, n_{z}, \ldots, n_{k}$. On a ici évidemment $m>n_{1}=1$ et il existe un nombre naturel $i \leq k$ tel que $m_{1}+m_{2}+\ldots+m_{i-1}<m<n_{1}+n_{2}+\ldots+n_{i}$. Va la condition que notre suite remplie, on a done $-1 \leq n_{1}+n_{2}+\ldots+n_{i-1}-n_{i}$ $<m-n_{i}<n_{1}+n_{2}+\ldots+n_{i_{-}}$. On a donc $m-n_{i} \geq 0$ et comme. d'après 
la définition du nombre $m$ il ne peut pas être $m-n_{i}=0$, le nombre $m-n_{i}$ est naturel et $<n_{1}+n_{2}+\ldots+n_{i-1}$ et comme, d'après $i-1<k$ notre condition est suffisante pour le nombre $i-1$, le nombre naturel $m-n_{i}$ est une somme de termes distincts de la suite $n_{1}, n_{2}, \ldots, n_{i-1}$, d'où il résulte que $m$ est une somme de termes distincts de la suite $n_{1}, n_{2}, \ldots, n_{i}$, contrairement à la définition du nombre $m$.

Notre condition est done suffisante. Nous avons ainsi démontré ce

THÉORÈME 2. $-n_{1}, n_{2}, \ldots, n_{k}$ étant une suite croissante de nombres naturels, la condition nécessaire et suffisante pour que tout nombre naturél $\leq n_{1}+n_{2}+\ldots+n_{k}$ soit une somme de termes distincts de cette suite est qu' on ait les inégalités (9).

Soit, en particulier, $n_{1}=1$ et $n_{i}=p_{l-1}$ pour $i=2,3, \ldots$, où $p_{i}$ désigne le $i$-ème nombre premier. Comme on le sait, on a

$$
p_{i+1} \leq 1+p_{1}+p_{2}+\ldots+p_{i} \text { pour } i=1,2, \ldots
$$

d'où il résulte que les nombres $n_{i}(i=1,2, \ldots)$ satisfont aux conditions (9) pour $i=1,2, \ldots$. Il résulte done tout de suite du théorème 2 ce

CoRollaire. - Tout nombre naturel est une somme de nombres distincts de la suite infinie $1, p_{1}, p_{2}, \ldots$, où $p_{i}$ est le i-ème nombre premier.

Or, il est à remarquer que M. H. E. Riohert a démontré en 1949 que tout nombre naturel $>6$ est une somme de nombres premiers distincts $\left({ }^{2}\right)$.

Il résulte tout de suite du théorème 2 que si $m$ est un nombre naturel tel que $m \leq n_{1}+n_{2}+\ldots+n_{k}$ et si $n_{j}$ est le plus grand des nombres $n_{1}$, $n_{2}, \ldots, n_{k}$ tel que $n_{j} \leq m$, le nombre $m$ est une somme de termes distincts de la suite $n_{1}, n_{2}, \ldots, n_{j}$ et on a $m \leq n_{1}+n_{2}+\ldots+n_{j}$.

Soit maintenant $j_{1}$ le plus grand indice $\leq k$ tel que $n_{j_{1}} \leq m$. On a donc $n_{j_{1}} \leq m \leq n_{1}+n_{2}+\ldots+n_{j_{1}}$ et si $m \neq n_{j_{1}}$, on trouve $0<m-n_{j_{1}} \leq n_{1}+n_{2}$ $+\ldots+n_{j_{1}-1}$ et si $j_{i} \neq 1$, il existe le plus grand indice $j_{2} \leq j_{1}-1$ tel que $n_{j_{2}} \leq m-n_{j_{1}}$ et on a $m-n_{j_{1}} \leq n_{1}+n_{2}+\ldots+n_{j_{2}}$, d' où si $m \neq n_{j_{1}}+n_{j_{2}}$ on trouve $0<m-n_{j_{1}}-n_{j_{2}} \leq n_{1}+n_{2}+\ldots+n_{j_{2}-1}$ et si $j_{2} \neq 1$, il existe le plus grand indice $j_{3} \leq j_{2}-1$, tel que $n_{j_{3}} \leq m-n_{j_{1}}-n_{j_{2}}$. En raisonnant ainsi de suite on arrive à la décomposition $m=n_{j_{1}}+n_{j_{2}}+\ldots$, où $k \geq j_{1}>j_{2}>\ldots$.

Voici encore une conséquence immédiate du théorème 2 :

ThÉoRime 3. - Pour qu' un nombre naturel $n$ soit un nombre pratique, il faut et il suffit qu'on ait pour la suite croissante de tous les diviseurs naturels $1=d_{1}, d_{2}, \ldots ; d_{s}=n d u$ nombre $n$ les inégalités

$$
d_{i} \leq 1+d_{1}+d_{2}+\ldots+d_{i_{-1}} \text { pour } i=1,2, \ldots, s .
$$

(2) H. E. Rrohert, * Norsk Matematisk Tidskrift ", 31 (1949), p. 120. 
Il résulte aussi du théorème 2 que si le nombre naturel $n$ remplit ces conditions, tout nombre naturel $m$ tel que pour un indice $i \leq k$ on a

$$
m \leq d_{1}+d_{2}+\ldots+d_{i}
$$

est une somme des nombres distinets de la suite $d_{1}, d_{2}, \ldots, d_{i}$.

Il en résulte la méthode suivante, signalée par M. SAROJA dans sa note citée, de déterminer pour un nombre naturel $m \leq 1+\sigma(n)$, où $n$ est un nombre pratique, la décomposition du nombre $m$ en une somme de diviseurs distincts du nombre $n$. On détermine le plus grand diviseur $d_{j_{1}}$ de $n$ tel que $d_{j_{1}} \leq m$. Si $m \neq d_{j_{1}}$, on détermine le plus grand diviseur $d_{j_{3}}<d_{j_{1}} \mathrm{du}$ nombre $n$ tel que $d_{j_{2}} \leq m-d_{j_{1}}$. Si $m \neq d_{j_{1}}+d_{j_{2}}$, on détermine le plus grand diviseur $d_{j_{3}}<d_{j_{2}}$ de $n$ tel que $d_{j_{3}} \leq m-d_{j_{1}}-d_{j_{2}}$ et ainsi de suite, jusqu'on arrive à la décomposition $m=d_{j_{1}}+d_{j_{2}}+\ldots+d_{j_{r}}$, où $j_{1}>j_{2}>\ldots>j_{r}$. 